\title{
Investigating a non-destructive alternative for a preliminary evaluation of fungal growth in solid state fermentations
}

DOI:

10.1016/j.mimet.2019.03.021

\section{Document Version}

Accepted author manuscript

Link to publication record in Manchester Research Explorer

\section{Citation for published version (APA):}

López-Gómez, J. P., Pérez-Rivero, C., \& Webb, C. (2019). Investigating a non-destructive alternative for a preliminary evaluation of fungal growth in solid state fermentations. Journal of Microbiological Methods, 160, 6067. https://doi.org/10.1016/j.mimet.2019.03.021

\section{Published in:}

Journal of Microbiological Methods

\section{Citing this paper}

Please note that where the full-text provided on Manchester Research Explorer is the Author Accepted Manuscript or Proof version this may differ from the final Published version. If citing, it is advised that you check and use the publisher's definitive version.

\section{General rights}

Copyright and moral rights for the publications made accessible in the Research Explorer are retained by the authors and/or other copyright owners and it is a condition of accessing publications that users recognise and abide by the legal requirements associated with these rights.

\section{Takedown policy}

If you believe that this document breaches copyright please refer to the University of Manchester's Takedown Procedures [http://man.ac.uk/04Y6Bo] or contact uml.scholarlycommunications@manchester.ac.uk providing relevant details, so we can investigate your claim.

\section{OPEN ACCESS}


Investigating a non-destructive alternative for a preliminary evaluation of fungal growth in solid state fermentations

\author{
José Pablo López-Gómez ${ }^{a, b *}$, Cristina Pérez-Rivero ${ }^{a}$ and Colin Webb ${ }^{a}$.
}

${ }^{a}$ School of Chemical Engineering and Analytical Science, The University of Manchester, Oxford road, Manchester, M13 9PL, United Kingdom.

${ }^{b}$ Department of Bioengineering, Leibniz Institute for Agricultural Engineering Potsdam-Bornim, Potsdam, Germany

*Corresponding author.

Email:plopezgomez@atb-potsdam.de

Tel: $+49(0331) 5699$ ext-857

\title{
Abstract
}

Solid state fermentation (SSF) is an ancient technique which keeps attracting the attention of the food and biotechnology industries; however, a direct quantification of microbial biomass is still a fundamental challenge in this type of processes. Typically, growth is measured using indirect and destructive methods which do not allow a continuous evaluation of the evolution of microbial biomass within a single system. This article presents a non-destructive, quick and simple technique, based on digital imaging analysis (DIA) for the evaluation of growth in SSF laboratory experiments. DIA uses computational analysis of images from a SSF to measure areas and colour changes on a surface. The method can then be used to monitor microbial growth by assigning quantitative values for the growth of filamentous fungi. Firstly, studies on agar plates are used for the description of the method and to illustrate how it can be used to monitor fungal colony areas and densities. Following that, agro-industrial residues are used to demonstrate the application of the technique. DIA proved to be a practical and inexpensive tool to measure colony areas and densities. Furthermore, it is a non-destructive and non-intrusive method, which means that the evaluation of growth can be achieved within a single system.

Keywords: solid state fermentation, fungal growth, digital imaging analysis, colour mean. 


\section{Introduction}

36

37

The intrinsic complexity of solid state fermentation (SSF) makes its control and application very difficult (Mitchell and Berovic, 2014) and, although experiencing growing interest, the evolution of SSF technologies has been rather slow. In the words of Mitchell et al. (1990) "Studies are hampered by the heterogeneity and complexity of the system and by problems in measuring fermentation parameters, the most critical of which is the biomass". In SSF, the microorganism attaches to the solid substrate and, more specifically for the case of filamentous fungi, the mycelia penetrate the solid particles making an effective separation very difficult if not impossible (Pandey et al., 2008). The heterogeneity of the substrate and the substrate/fungal amalgam, together with the inability to perform the separation between the substrate and the microbe, make a direct quantification of microbial growth an unsolved fundamental problem for such processes (Manan and Webb, 2018a; Simeng et al., 2015; Steudler and Bley, 2015).

Since a direct quantification of growth is not possible some indirect methods, based on measuring biomass components, have been developed (Mitchell et al., 2000). Commonly, the estimation is performed by quantifying specific components of fungi such as ergosterol, glucosamine, nucleic acids, proteins, fungal specific phospholipid fatty acids and spores (Steudler and Bley, 2015). However, one of the downsides of this type of measurement is that the relationship between cell components and fungal biomass may not be constant with time (Davey et al., 1991; Mitchell et al., 2000). Thus, although widely used in practice, results from such measurements only provide, at best, biomass estimates rather than absolute values of growth (Mitchell et al., 2004). Furthermore, such measurements require the homogenization of the samples during preliminary laboratory experiments. However, sacrificing whole cultures every time a measurement is required makes it impossible to follow the progress of a SSF in a single system and numerous replicates are necessary for the evaluation of growth, which makes the procedures destructive and impractical (Desgranges et al., 1991). 
Colour measurement is widely used in many fields as a parameter that can be related to other

61 properties of interest in a system. During growth on a solid substrate, a fungal colony expands while its mycelia density increases. As a result, there is an apparent variation in the colour of the substrate/fungal biomass mixture. Ramana Murthy et al., (1993), performed an investigation in which the change in colour of ground substrate, monitored using light reflectance, was an indicator of biomass presence. They compared the results from colour measurement to those from glucosamine and concluded that there was a good agreement between the different methods of quantification. Manan and Webb (2018b, 2016), reported a study based on spectrophotometer measurements of extracts from SSF systems to monitor the growth of fungi. They compared the results from the colour density measurements with those from enzyme production (as an indicator of growth) and concluded that there was good agreement between the methods. Nevertheless, as already mentioned, in addition to the length of the procedures, a drawback of both the above methods is still the need to sacrifice a plate every time a measurement is performed.

Colour measurement has also been studied as a method to identify fungal cultures on agar plates (Dörge et al., 2000), however, to the best of the authors' knowledge, direct measurement of colony colour for the evaluation of fungal growth in SSF has never been reported. The situation discussed above motivated the research reported in this article, to develop and investigate a quick and simple technique that could provide estimates of fungal biomass without the disadvantages of the more common alternatives. A novel idea based on the monitoring of colour variation in the SSF using digital images is presented.

\subsection{Description of the method}

82 Commonly, preliminary lab SSF experiments are carried out in petri dishes. In such cases, an alternative approach for the evaluation of growth is the measurement of colony areas in which larger areas indicate better growth (for cases where microbial growth is evaluated in agar media)

85 (Brancato and Golding, 1953; Couri et al., 2006; Li and Wadsö, 2011; Mitchell et al., 1988). In 
this type of studies, a regular colony shape used to be critical to simplify the task of measuring areas (Reeslev and Kjøller, 1995). Nowadays, with the development of better software and digital imaging systems, it is possible to perform the measurement of the areas, regardless of their shape, in a quick and simple way (Li and Wadsö, 2011). Athough a good indicator of growth, the measurement of area occupied by the colony is not sufficient to evaluate fungal biomass production (Bull and Trinci, 1977; Mitchell et al., 1988). It is possible that colonies of the same areas differ in fungal biomass, due to variations in their colony density.

The histogram (Figure 1) is a fundamental component of any image manipulation software. It provides valuable information that allows the quantification of each colour in an image. In a histogram, the $\mathrm{x}$-axis shows the intensity of the colour, which goes from 0 to 255 , and the $y$-axis shows the number of pixels with that intensity. This feature can be used and exploited for the quantification of colour intensities of red, blue and green in an image. Computational software can calculate an average of the intensity of the colours and translate the final colour into a numerical value which corresponds to the mean.

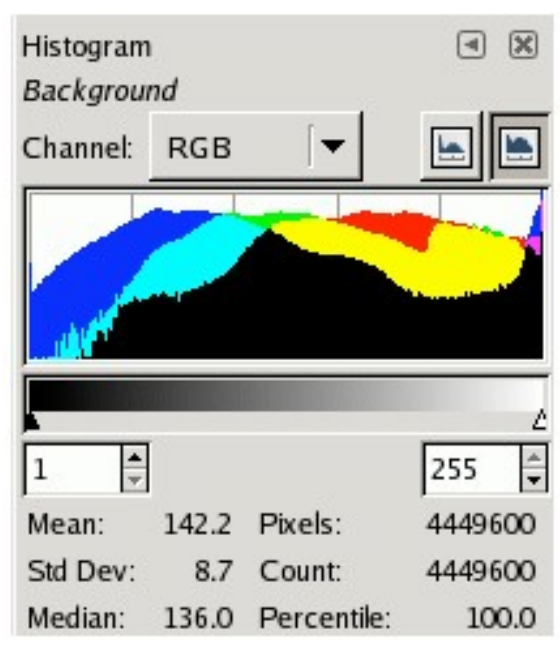

Figure 1: Image of the histogram obtained in GIMP software. It can be obtained from any image (or part of it) and provides information of the number of pixels contained as well as the colour mean (mean) value.

In any plate, the growth of fungi would either reduce or increase the colour mean value. For the case of fungi with black spores, such as A. awamori, the colour mean should decrease as the 

very quick and simple.

Figure 2 exemplifies how digital imaging analysis could be used to evaluate fungal growth. The image simulates areas that, although having the same size, differ in fungal density. In this case, larger values represent a lesser dense colony than those from the lower values. In the example, a plate without growth would have a value of 255 and a plate completely covered by fungi, would have a value of 0 .

A

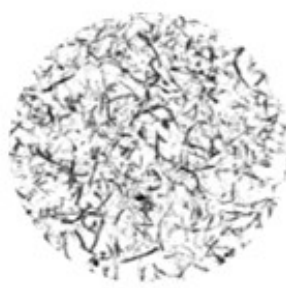

208.6

\section{B}

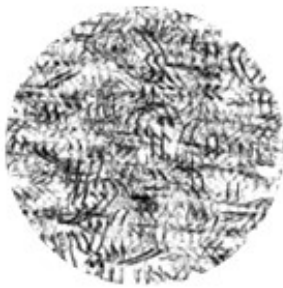

170.6

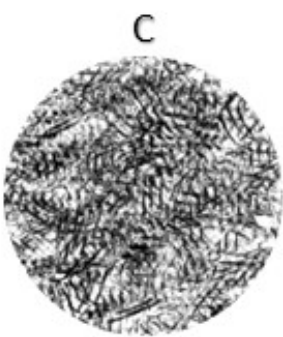

140.2

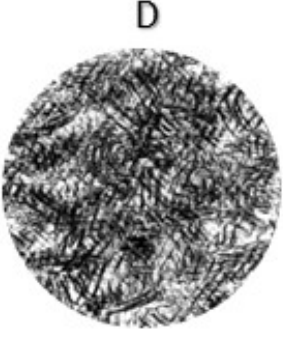

115.6

Figure 2: Schematic representation of the variation of colony densities in areas of the same size. The values presented indicate the colour mean (measured using GIMP) for each plate. A value of 255 represents total brightness (white) and a value of 0 represents total darkness (black).

In contrast to a greyscale image, in which a pixel can only acquire 256 values (corresponding to the intensity of the pixel with values from 0 to 255), a pixel in a colour image includes values for intensities of red, blue and green or $256 * 256 * 256$ which results in a total of $16,777,216$ possible combinations, drastically increasing the sensitivity of the measurement. Due to their lower complexity and storage requirements, greyscale images tended to be preferred over colour images for solving mathematical models while saving space. Nonetheless, with the improvement in computing storage systems, the file sizes are not anymore a problem. Furthermore, in some cases, a measurement in colour or colour variations is indeed what really matters. The addition of an extra step to convert the colour image into grayscale does not bring any extra information but the opposite, reduces the info that we can extract from the photo. Due 

et al., 2000; Plavcan, 2004; Stevens et al., 2007).

\section{Materials and methods}

\subsection{Inoculum preparation}

133

The microorganism, $R$. oryzae NRRL 395 was obtained from LGC Standards, Teddington, United Kingdom. A. awamori, was obtained from the School of Chemical Engineering and Analytical Sciences, in the Faculty of Sciences \& Engineering of The University of Manchester. The organisms were cultivated, following the method described in (López-Gómez et al., 2015), on petri dishes with potato dextrose agar (PDA) at $30^{\circ} \mathrm{C}$. After 6 days, spores of the fungi were collected with $10 \mathrm{~mL}$ of a sterile Tween $80(0.01 \% \mathrm{v} / \mathrm{v})$ solution. Spore suspensions, with approximately $2 \times 10^{6}$ spores $/ \mathrm{mL}$, were obtained, poured into universal bottles and stored at $4^{\circ} \mathrm{C}$.

\subsection{Digital imaging analysis (DIA)}

Images of the samples were taken using a NIKON D3200, the set up of the camera and samples is shown in Figure 3. General Image Manipulation Program (GIMP), is a free and open-source raster graphics editing software and was used for the analysis of colony occupation areas and measurement of colour intensity in the images. Lighting conditions have an effect on the colour intensities of an image; therefore, it was necessary to take the images in a closed room with artificial light (without natural variations of light). Measurements of the colour mean using GIMP were performed without making any modification to the images. 


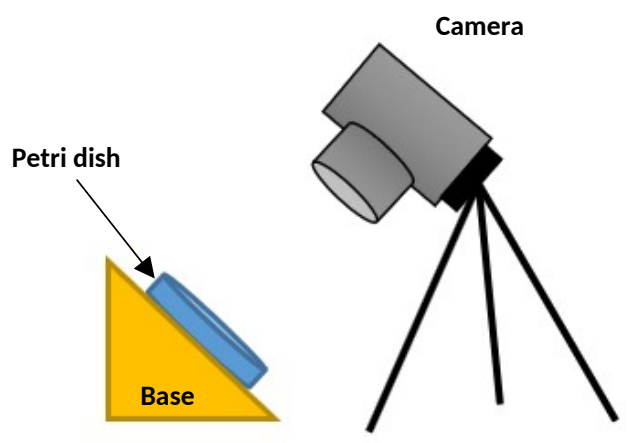

150

Figure 3: Camera and sample set up for the capture of the images of growth in petri dishes during SSF.

\subsection{Studies on agar plates}

In a first step, studies on agar plates were carried out to evaluate the feasibility of measuring colour intensity. Potato dextrose agar plates were prepared, inoculated (at the centre of the plate) with $10 \mu \mathrm{L}$ of a $R$. oryzae spore suspension $\left(2 \times 10^{6}\right.$ spores $\left./ \mathrm{mL}\right)$ and incubated at $30^{\circ} \mathrm{C}$. Growth of the fungus was followed and images of the plates were taken at different time intervals.

Measurements of the colour mean were performed in three different delimited areas of the plate. The first one, the total area, corresponds to the full plate. The second, occupied area, corresponded to the section of the plate covered by the fungal mycelia. The third one, origin area, corresponded to a circular section $\left(1.3 \mathrm{~cm}^{2}\right)$ with origin at the centre of the plate. Finally, a section from an image of a plate after $45 \mathrm{~h}$ of incubation was analysed. The section, which covered an area from the origin of the petri dish to its edge, was further divided into subsections and their colour mean values were measured.

\subsection{Studies using sugarcane bagasse as a model solid support}

Sugarcane bagasse was used as a typical natural solid substrate for experiments. It was obtained, 
the substrate was ground using a kitchen blender. Following that, the solid particles were separated according to their size using a sieve. The particles with sizes in the range of 1400-850 $\mu \mathrm{m}$ were used for the experimentation. They were washed using copious amounts of tap water, to remove sugars and impurities, and after that placed in an oven at $60^{\circ} \mathrm{C}$ for 3 days. After drying, the material was stored in air-tight plastic containers until used.

Before the cultivations, the moisture content of the bagasse was adjusted to $76 \%$ by mixing the dried substrate with sterile water or solutions containing different concentrations of yeast extract. The resulting sugarcane bagasse samples had a final concentration of $0,10,20,25$ and $40 \%(\mathrm{w} / \mathrm{w})$ of yeast extract. Petri dishes were prepared (in triplicate) with $1.5 \mathrm{~g}$ of the samples.

Samples with concentrations of 0,10 and $20 \%$ of yeast extract were inoculated by mixing $400 \mu \mathrm{L}$ of an A. awamori spore suspension $\left(2 \times 10^{6}\right.$ spores $\left./ \mathrm{mL}\right)$ into each sample. Samples with concentrations of 10, 25 and $40 \%$ were inoculated at the centre of the plate with same inoculum volume and concentration. After the inoculation, petri dishes were placed inside an incubator at $30^{\circ} \mathrm{C}$. Finally, digital images were taken after 4 days of incubation.

\subsection{Studies using an industrial residue}

Industrial residues, namely, stillage and wheat bran, were obtained from the wheat based ethanol production plant of Cargill PLC., Trafford Park, Manchester, UK. In this plant, the residues are combined to produce moist feed for cattle. The stillage and the wheat bran were stored (for a maximum of 2 weeks) in sealed plastic bottles in a cold room at $4^{\circ} \mathrm{C}$. The moist feed substrate was prepared by mixing wheat bran $(34 \% \mathrm{w} / \mathrm{w})$ and stillage $(66 \% \mathrm{w} / \mathrm{w})$. Initial water content of the moist feed was $50 \% \mathrm{w} / \mathrm{w}$.

Samples of the moist feed with moisture contents of $45,50,55,60$ and $65 \%$ were prepared. The required amount of sterile distilled water was added to the stillage, before mixing it with the wheat bran, for the preparation of the samples at 55, 60 and $65 \%$ moisture content. For the case 
of the sample with $45 \%$ moisture content, the stillage was dried in an oven at $60^{\circ} \mathrm{C}$ until its moisture content was reduced from 70 to $63 \%$, before being mixed with the wheat bran.

The inoculation was performed, using a spore suspension of $R$. oryzae, in the stillage before it was mixed in with the wheat bran, to achieve a spore concentration of approximately $10^{4}$ spores $/ \mathrm{g}_{\text {moist feed }}$. Following that, triplicates containing approximately $20 \mathrm{~g}$ of the moist feed samples were prepared. The plates were incubated at $20^{\circ} \mathrm{C}$ and pictures were taken at different times for colour intensity measurement using GIMP.

\section{Results and discussion}

\subsection{Studies on agar plates}

Figure 4 shows the average values for the colour mean from triplicates of the three different areas of the plates. At $\mathrm{t}=0 \mathrm{~h}$, right after the inoculation, the average colour mean of the plates was 50.1. At $\mathrm{t}=20.5 \mathrm{~h}$, the values for the colour mean of the origin area $\left(1.3 \mathrm{~cm}^{2}\right)$ were larger than those of the total area. This difference indicates that the inoculum causes an increase in the colour mean value which shows that it is brighter than the background. In this particular case, an increase on the colour mean values over time indicates an increase in biomass.

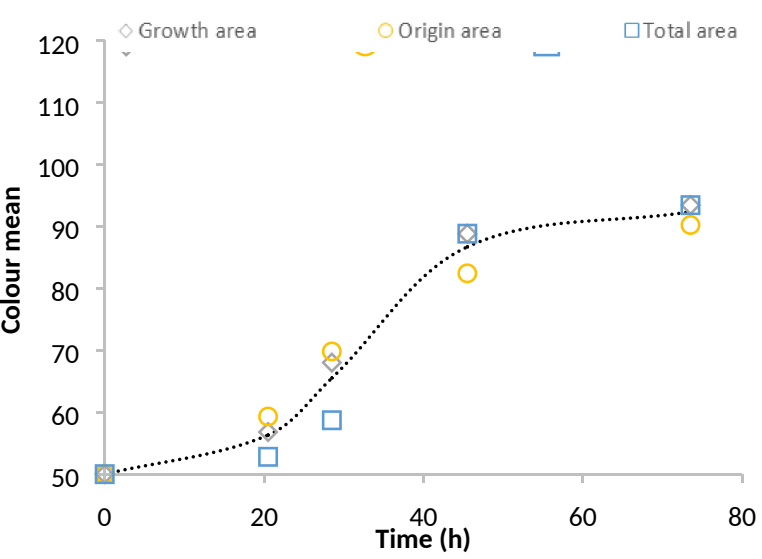

Figure 4: Evolution of growth in a PDA plate inoculated with $10 \mu \mathrm{L}$ of a $R$. oryzae spore suspension. Fungal density and colony occupation areas vary with time. The colour mean for all the sections increased with time resulting in a sigmoidal-shaped curve. The dotted line is the average of the three curves. 
213 Although all the colour mean values increased, as observed in the figure, there were differences

214 between the origin area and the occupied area which indicated a difference in fungal density

215 within the colony. By $\mathrm{t}=45.5 \mathrm{~h}$ the colony already occupied all the plate hence the value for the

216 occupied area remained constant $(100 \%)$ after that time. Nevertheless, a change on the colour

217 mean values could still be observed after that, indicating a change in fungal density.

218 Figure 5, shows a close-up of a section of a plate at $45.5 \mathrm{~h}$ of incubation. As seen in the figure,

219 there are colour variations within the colony.

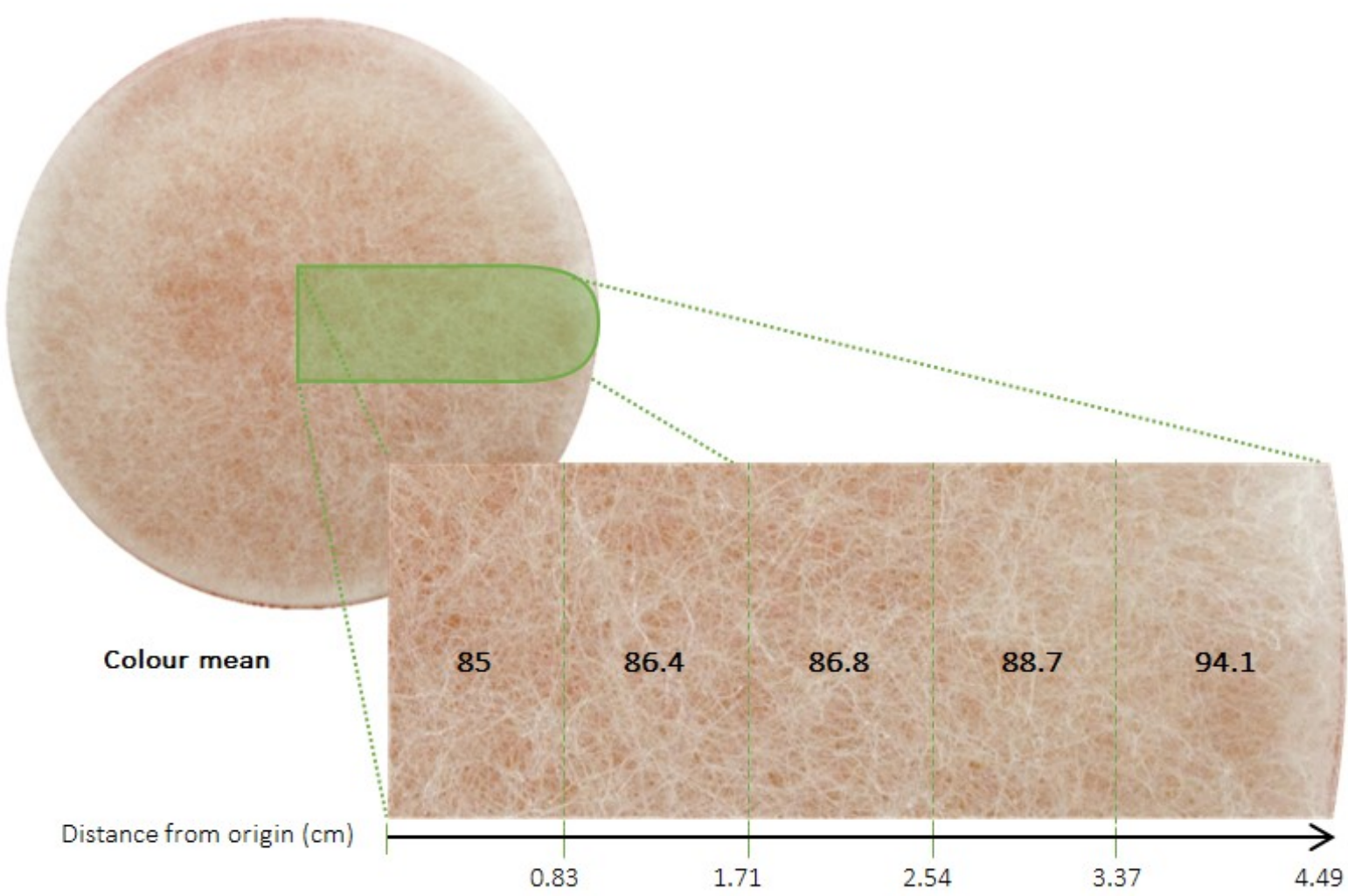

Figure 5: Difference of colony density in terms of colour mean in a PDA plate inoculated with a $R$. oryzae spore suspension. Colour mean values suggest that sections of the colony closer to the edge of the plate showed a greater fungal density.

As seen in Figure 5, sections of the plate closer to the edge showed larger values for the colour mean than those near the centre of the plate. This difference was probably due to limitation of oxygen in the centre of the plate. Aeration in petri dishes occurs by diffusion and the oxygen that becomes available for the fungus enters the dish through the space between the dish and the 
lid. At $\mathrm{t}=0$, oxygen is readily available in all the plate and during the first $28.5 \mathrm{~h}$ the colour mean for the origin section goes from 50.1 to 69 . However, after the colony occupied the outer sections of the petri dish, the variation in the colour mean for the origin section became slower, reaching 82.4 only after $45 \mathrm{~h}$. In contrast, at the same time, 'younger' sections of the colony (nearer the edge of the plate) showed a colour mean value of already 94.1 and a maximum value of around 120 for some areas.

\subsection{Combination of fungal density and occupation areas}

236 A petri dish undergoes a change in its colour mean from the colour of the substrate (or 237 background) at $\mathrm{t}=0$ to that of the microorganism as growth occurs and the colony becomes denser. When measuring colony densities there should be a maximum or minimum value for the colour of the colony which represents the maximum density of the colony, i.e. the actual value for the colour of the microorganism under the specific lighting environment. In the given example this value is 120 . Although in the colour histogram the scale goes from 0 to 255 , there are certain colour values (x-axis) that are empty. In the example discussed above, the minimum value that the colour mean had was 50 (from the background) and the maximum value was 120 .

244 The difference between the maximum and minimum colour mean values, i.e. the effective colour range of the image $\left(\Delta \bar{x}_{R G B}\right)$ can be used as a reference to determine the relative change in colour of one sample.

247

$$
\begin{gathered}
\Delta \overline{\mathrm{x}}_{\mathrm{RGB}}=\overline{\mathrm{x}}_{\mathrm{RGB}, \max }-\overline{\mathrm{x}}_{\mathrm{RGB}, \min } \\
\mathrm{K}_{\mathrm{RGB}}=\frac{\left|\overline{\mathrm{x}}_{\mathrm{RGB}, \mathrm{i}}-\overline{\mathrm{x}}_{\mathrm{RGB}, \text { initial }}\right|}{\Delta \overline{\mathrm{x}}_{\mathrm{RGB}}} \times 100
\end{gathered}
$$


254 The values for $K_{R G B}$ can go from $0 \%$ (no growth) to $100 \%$ (maximum colony density). In the previous example, a sample with a colour mean value of 120 has a $K_{R G B}$ equal to $100 \%$ which indicates that it has achieved the maximum density value possible for the colony. A sample with a colour mean value of 50 has a $K_{R G B}$ equal to $0 \%$ which indicates that no growth has occurred. A comparison of the $K_{R G B}$ and colour mean curves for the experiment is shown in Figure 6.

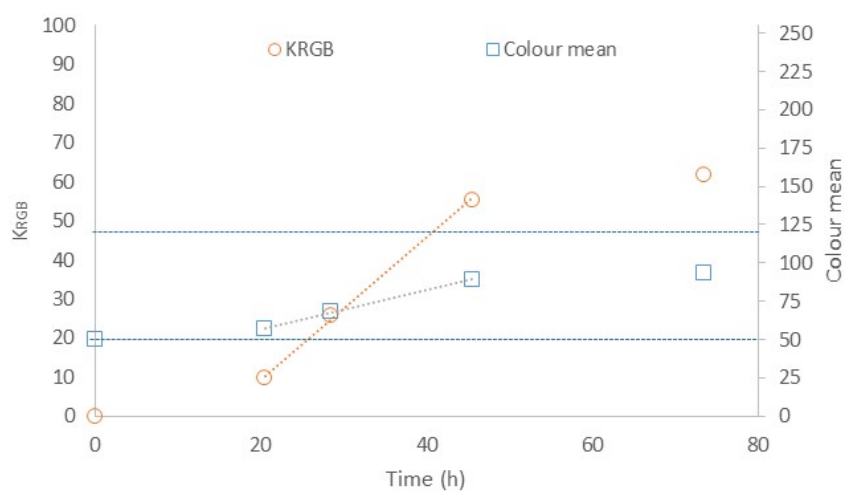

Figure 6: Comparison between the colour mean and $K_{R G B}$ curves. As observed, the curve for $K_{R G B}$ shows a more pronounced change than the colour mean curve. The blue dotted lines are the upper and lower limits of the colour mean (120 and 50$)$.

263 The advantage of $K_{R G B}$ is that it provides a clearer indication of how the process progresses.

264 Since the values relate to the colour mean values that a colony has reached, and not to the full colour scale, it is possible to evaluate the growth.

\subsection{Studies using sugarcane bagasse as a model solid support}

267 Experimental work was performed to illustrate how DIA could be used for the evaluation of 268 growth in SSF on natural substrates. In the experiments, sugarcane bagasse was used as the solid support and carbon source for the growth of A. awamori. The substrate was supplemented with a nitrogen source (yeast extract) in varying ratios and results after 4 days of incubation are shown in Figure 7. 

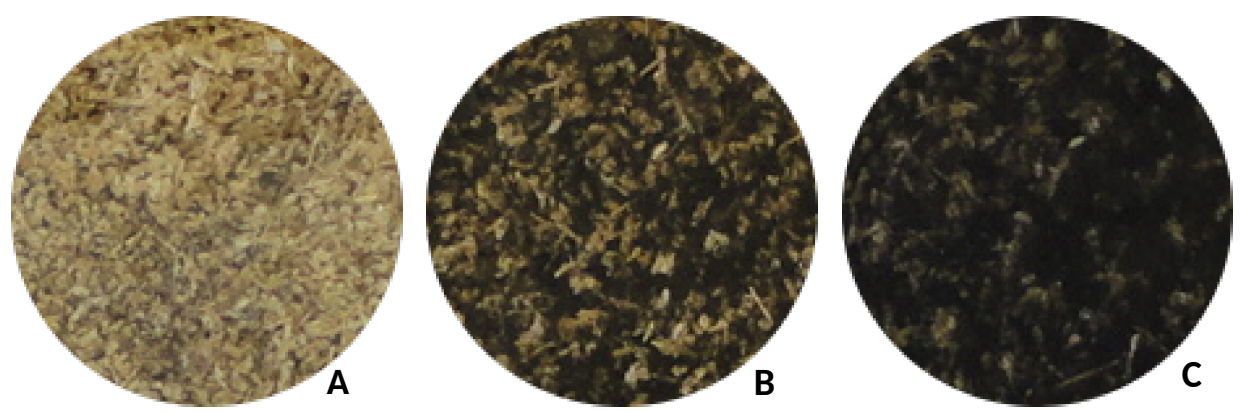

\begin{tabular}{|c|ccc|}
\hline Sample & A & B & C \\
\hline Yeast extract & $0 \%$ & $10 \%$ & $20 \%$ \\
\hline Colour mean & 130 & 53.5 & 32.3 \\
\hline
\end{tabular}

Figure 7: Pictures taken after 4 days of a SSF on sugarcane bagasse mixed with yeast extract in different ratios. As observed, the quantification of colour mean values allows a rapid estimation of growth in the plates.

277 From the figure it would be possible, just visually, to define that growth in sample A is less than

in sample B, which is less than in C. Although useful, that method of comparison does not give

a clear indication of the degree of difference between samples. Differences in the values for the colour mean perhaps provide a better indicator of growth.

A similar experiment was performed in which the samples were first placed into petri dishes and then the inoculation was performed in the centre of the plate. Figure 8 shows the results for the colour mean and colony occupation areas performed at 42,66 and $91 \mathrm{~h}$. The colour mean values are those corresponding only to the occupied area of each sample.
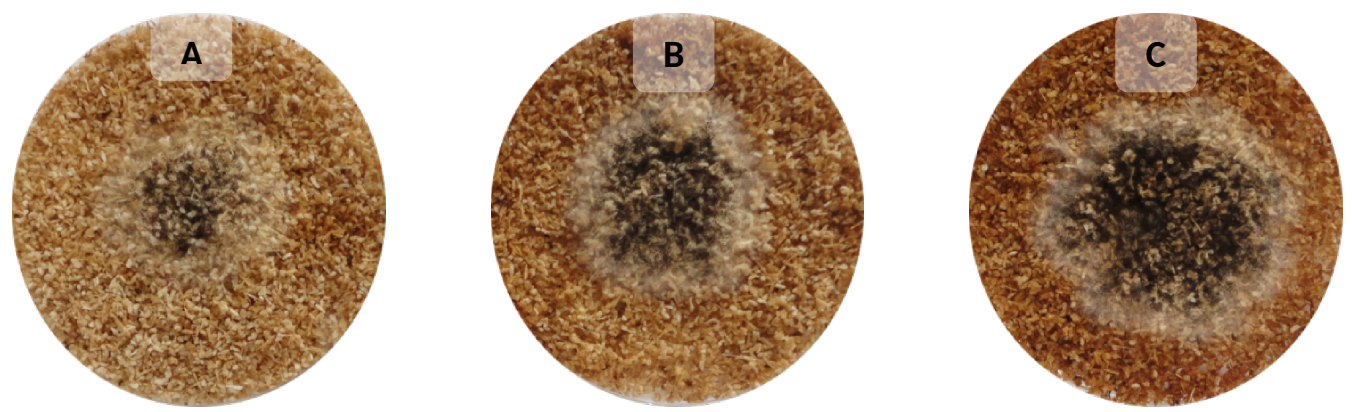

\begin{tabular}{|c|cc|cc|cc|}
\hline \multirow{2}{*}{ Yeast extract } & \multicolumn{2}{|c|}{$42 \mathrm{~h}$} & \multicolumn{2}{c|}{$66 \mathrm{~h}$} & \multicolumn{2}{c|}{$91 \mathrm{~h}$} \\
\cline { 2 - 7 } & Area (\%) & Colour mean & Area (\%) & Colour mean & Area (\%) & Colour mean \\
\hline A $(10 \%)$ & 18 & 78 & 39 & 56 & 48 & 45 \\
B (25\%) & 25 & 71 & 45 & 38 & 66 & 32 \\
C (40\%) & 40 & 60 & 63 & 37 & 82 & 23 \\
\hline
\end{tabular}


Figure 8: Effect of yeast extract on the growth of A. awamori on sugarcane bagasse. Images of the systems after $42 \mathrm{~h}$ of incubation. As observed a higher yeast extract concentration has a positive effect on the colony size and on colour mean values.

290 Figure 8 shows the advantage of inoculating the plates in the centre rather than mixing the

291 inoculum with the substrate. Even without colour intensity measurements it is clear the effect

292 that the $\mathrm{N}$ source has on the growth in terms of radius of the colonies. The plate with lower concentration of $\mathrm{N}$ produced the smallest occupied area $(18 \%)$ whereas the plate with more $\mathrm{N}$ showed the largest area $(40 \%)$. However, the difference between fungal densities is not obvious, unlike in Figure 9 where even without DIA it is possible to observe that growth density is greater as the yeast extract content increases.

297

298
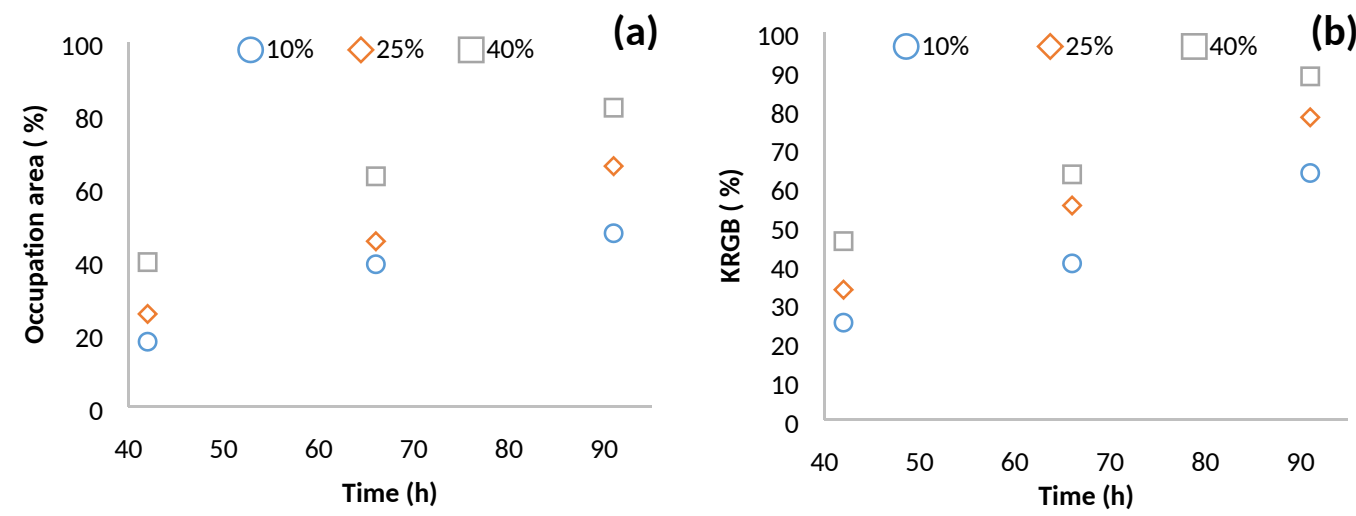

(b)

Figure 9: Effect of yeast extract concentration on colony occupation areas (a) and $K_{R G B}$ (b). $K_{R G B}$ values were calculated based on $\bar{x}_{R G B, \min }=13$ and $\bar{x}_{R G B, \max }=100$.

Figure 9a gives an indication of how fast the colonies expand on the plates. For example, in the figure, each sample contains a data point at around $40 \%$ colony occupation area. Although it is possible to state that as the yeast extract content increases the colony expansion rate increases, it is not possible to determine which colony is denser when they reach around $40 \%$ occupation area. Conversely, while the values for $K_{R G B}$ (Figure 9b) give an indication of colony density, they do not distinguish colony size. By combining both measurements a much more complete picture of growth can be obtained (Figure 10). 


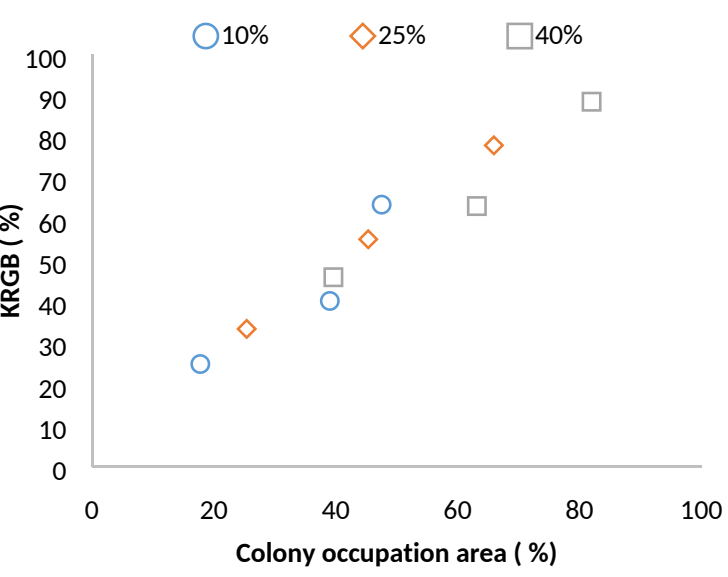

307

308

309

310

311

312

313

314

315

316

317

$$
K_{D I A}=\frac{a_{g} x K_{R G B}}{100}
$$

319

320

321

322

323 $\bar{x}_{R G B, \min }=13$ and $\bar{x}_{R G B, \max }=100$. $40 \%$ yeast extract case. growth' for the evaluation of growth:

Figure 10: Relationship between $K_{R G B}$ and occupation areas. $K_{R G B}$ values were calculated based on

Such a graph can be used to compare growth in samples taking into consideration how large and dense fungal colonies are. In the graph, data points that approximate $100 \%$ indicate better growth. Let us consider again the case of occupation areas of almost the same size $(39.02 \%$, $39.60 \%$ ). Even though both values for the colour mean are similar, the data point for the sample with more yeast extract has a higher $K_{R G B}$. Similarly, two data points with the same $K_{R G B}$ (65\%) showed different occupation areas (47\% and $63 \%)$ indicating better growth for the

Values from Figure 10 can be used to calculate a 'Digital imaging analysis coefficient of

\section{$K_{D I A}=$ Digital Imaging Analysis coefficient of growth $a_{g}=$ area of growth}

As observed, $\mathrm{K}_{\mathrm{DIA}}$ is a relationship between the occupation areas and colour mean i.e. colony density. $\mathrm{K}_{\text {DIA }}$ values for the samples were calculated and the results are shown in Figure 11. 


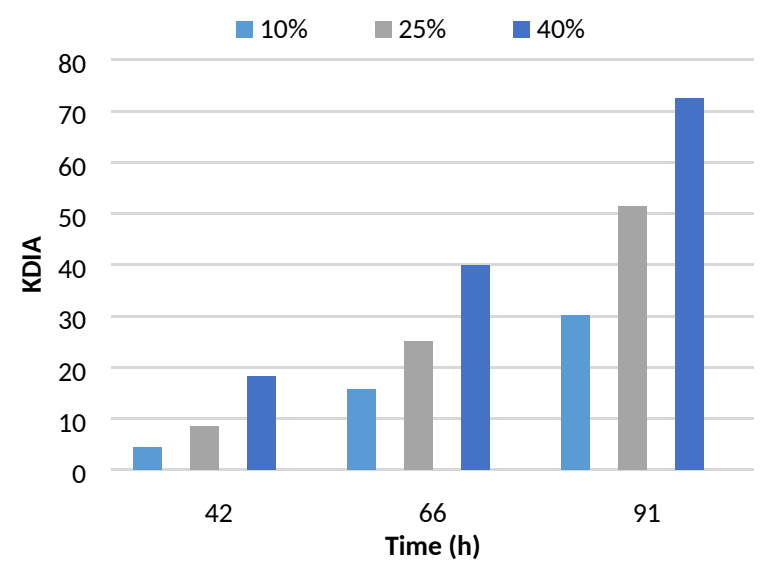

Figure 11: KDIA values for A. awamori grown on sugarcane bagasse supplemented with different concentrations of yeast extract.

\subsection{Studies using an industrial residue}

329 The positive effect that moisture content has on growth is clear in Figure 12.

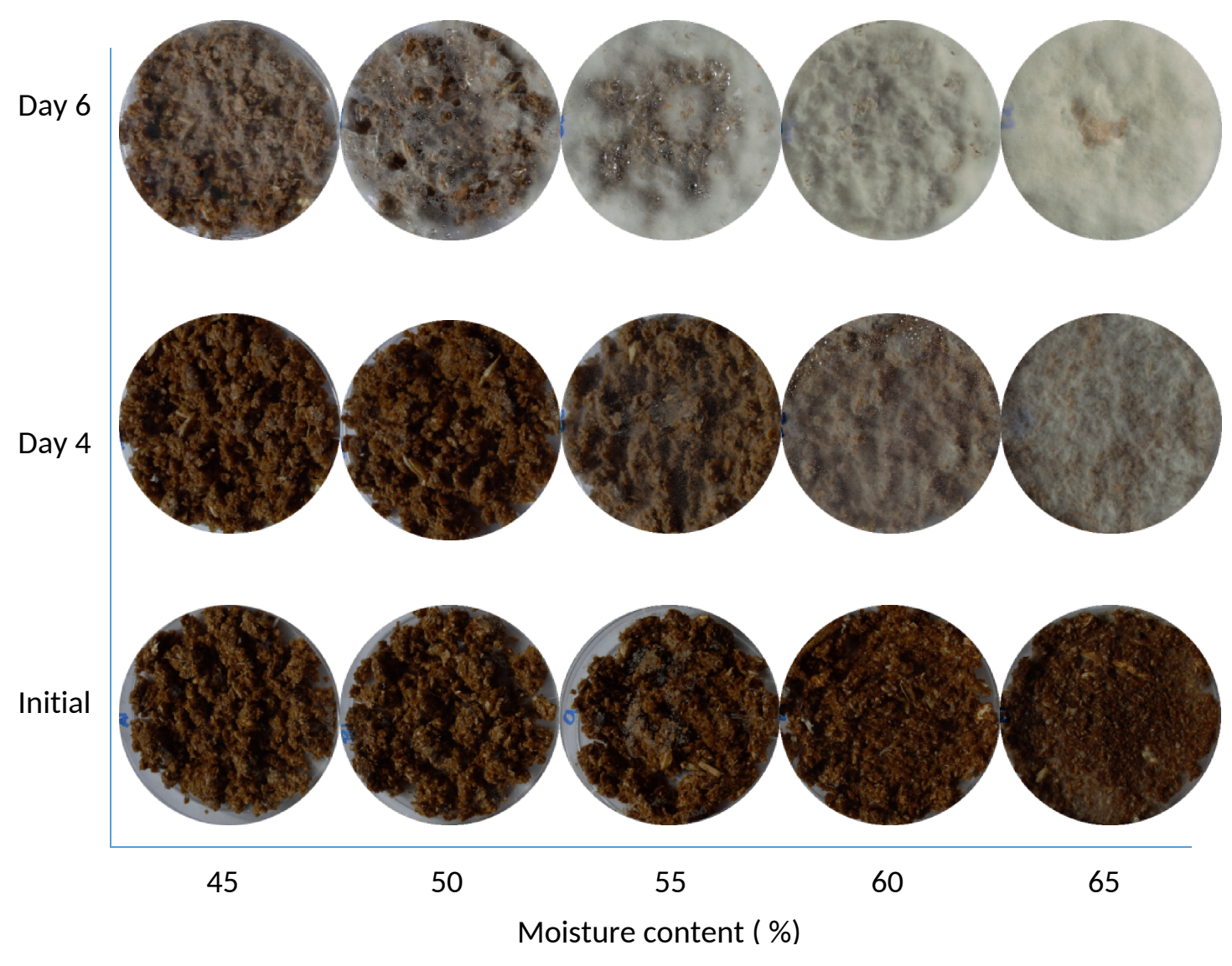



resulted in more apparent growth. The standard sample (50\% moisture content) was the second lowest with respect to growth. In addition to increasing the amount of growth, higher moisture contents also accelerated the appearance of visible colonies. Evidently, analysis of this type (qualitative) is very useful and can be further enhanced if DIA is applied as shown in Figure 13 (Values of $\bar{x}_{R G B, \max }=160$ and $\bar{x}_{R G B, \min }=35$ were used for the calculation of $\mathrm{K}_{\mathrm{RGB}}$ ).

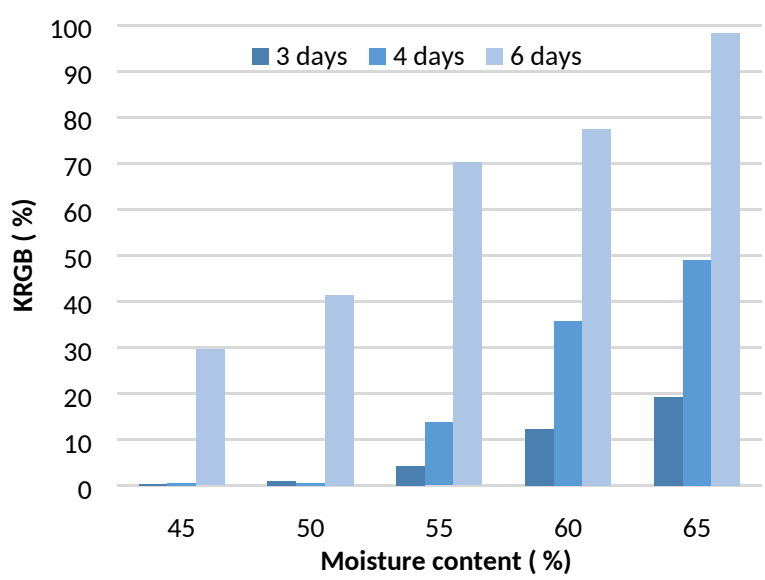

Figure 13: Effect of the moisture content on SSF of stillage and wheat bran mixture samples on $K_{R G B}$. Plates with higher moisture content showed better growth of $R$. oryzae and higher $K_{R G B} . K_{R G B}$ values were calculated based on $\bar{x}_{R G B, \min }=35$ and $\bar{x}_{R G B, \max }=160$.

The graph shows that increasing the moisture content not only benefits growth but also that there is an apparent linear relationship between these two variables for the samples tested. The sample with $65 \%$ moisture content had the highest $\mathrm{K}_{\mathrm{RGB}}$ at every sample time. At 6 days, the sample with $65 \%$ moisture content reached a $\mathrm{K}_{\mathrm{RGB}}$ of $98 \%$ while the sample with lowest moisture content (45\%) barely reached $30 \%$. Clearly, increasing the moisture content of the moist feed enhances the growth of $R$. oryzae. 
353 Digital imaging analysis (DIA) proved to be a practical tool that can be used to measure colony 354 areas and densities in a quick and simple way. Besides, it is a non-destructive non-intrusive method, which means that estimation of growth can be followed in the same sample. In situations where there are many samples to analyse, DIA can be easier to implement than other more common methods to estimate growth. The technique provides values that allow an objective comparison between plates, making it of higher quality than just visual evaluation. The parameter $\mathrm{K}_{R G B}$ (relative value of colour intensity change) is a normalised value that allows the evaluation of growth on a surface based on its colour change. This value allows the comparison between samples and also to determine how far a colony is from reaching its maximum colour density i.e. colony density. A combination between colony occupation areas and $\mathrm{K}_{R G B}$ was used to create $\mathrm{K}_{D I A}$ (Digital imaging analysis coefficient of growth). This coefficient strengthens the applicability of colony occupation areas because it also includes the density of the colonies. Hence, it is possible to compare colonies of the same occupation areas.

Evidently, DIA provides information only from the surface of a SSF system. Growth occurring in sections under the surface of the substrate cannot be monitored using this technique. This clearly is a limitation in systems where homogeneous growth throughout the substrate is desired. Nevertheless, because the surface is where oxygen is more readily available, growth on those areas should, in principle, occur at its best. Hence, DIA can be used in preliminary experiments in which the evaluation for the best conditions for growth is performed. Additionally, in systems where mixing occurs, microbial growth should produce colour intensity changes within the substrate. Therefore, in principle, the variation in colour intensity could be used also in that type of system. Probably, the analysis would include the determination of a maximum/minimum colour mean value (indicating best growth) which could be used for the evaluation of growth across the whole substrate bed. 
377 DIA is based on colour intensity variations, thus only fungal colonies with different colour

378 intensity from the substrate matrix would influence the change of colour intensity in an image.

379 That being said, the software is highly sensitive, and it is capable of detecting even small

380 differences. Further experimental work is necessary to determine the minimum difference in

381 colour intensities, between the substrate and the microorganism, necessary to achieve an

382 effective quantification. Future studies should focus on the applicability of DIA to more

383 complex SSF.

384

385

386

387

388

389

390

391

392

Additionally, costs associated with the technique are just those related to the camera and digital imaging software making it a very cost effective method. Moreover, the standardisation of aspects such as lighting environment, geometry of the sample, distance from the sample to the camera, measurement of initial colour intensity of the sample and so on, would allow an objective comparison between experiments carried out anywhere in the world. The possibility of developing automated systems coupling SSF and DIA would further enhance the value of the method.

\section{Acknowledgements}

The authors would like to thank the "National Council of Science and Technology" CONACyT and Cargill PLC for the financial support for this research.

\section{References}

Brancato, F.P., Golding, N.S., 1953. The Diameter of the Mold Colony as a Reliable Measure of Growth. Mycol. Soc. Am. 45, 848-864.

Bull, A.T., Trinci, P.J., 1977. The physiology and metabolic control of fungal growth., in: Advance in Microbial Physiology. Academic Press Inc, pp. 1-84.

Couri, S., Mercês, E.P., Neves, B.C. V, Senna, L.F., 2006. Digital image processing as a tool to monitor biomass growth in Aspergillus niger 3T5B8 solid-state fermentation: preliminary 
402

403

404

405

406

407

408

409

410

411

412

413

414

415

416

417

418

419

420

421

422

423

Davey, C.L., Peñaloza, W., Kell, D.B., Hedger, J.N., 1991. Real-time monitoring of the accretion of Rhizopus oligosporus biomass during the solid-substrate tempe fermentation. World J. Microbiol. Biotechnol. 7, 248-59. https://doi.org/10.1007/BF00328998

Desgranges, C., Vergoignan, C., Georges, M., Durand, A., 1991. Biomass estimation in solid state fermentation I. Manual biochemical methods. Appl. Microbiol. Biotechnol. 35. https://doi.org/10.1007/BF00184686

Dörge, T., Carste, Nsen, J.M., Frisvad, J.C., 2000. Direct identification of pure Penicillium species using image analysis. J. Microbiol. Methods 41, 121-133. https://doi.org/10.1016/S0167-7012(00)00142-1

Li, Y., Wadsö, L., 2011. Simultaneous measurements of colony size and heat production rate of a mould (Penicillium brevicompactum) growing on agar. J. Therm. Anal. Calorim. 104, 105-111. https://doi.org/10.1007/s10973-010-1251-5

López-Gómez, J.P., Blanco-Rosete, S., Webb, C., 2015. Extending shelf life of wheat based animal feed using solid state bioprocessing. Chem. Eng. Res. Des. https://doi.org/10.1016/j.cherd.2015.10.049

Manan, M.A., Webb, C., 2018a. Estimation of growth in solid state fermentation: A review. Malays. J. Microbiol. 14, 61-69.

Manan, M.A., Webb, C., 2018b. Colour changes as an indicator for estimating fungal growth in solid state fermentation. Malays. J. Microbiol. 14, 254-264.

Manan, M.A., Webb, C., 2016. Extracted substrate colour as an indicator of fungal growth in solid state fermentation $12,445-449$.

Mitchell, D. a., von Meien, O.F., Krieger, N., Dalsenter, F.D.H., 2004. A review of recent 
developments in modeling of microbial growth kinetics and intraparticle phenomena in solid-state fermentation. Biochem. Eng. J. 17, 15-26. https://doi.org/10.1016/S1369-

Mitchell, D.A., Berovic, M., 2014. Solid State bioprocessing, in: Principles of Bioprocessing Engineering. University of Ljubljana, pp. 244-276.

Mitchell, D.A., Berovic, M., Krieger, N., 2000. Biochemical Engineering Aspects of Solid State Bioprocessing. Adv. Biochem. Eng. Biotechnol. 68, 61-138.

431

432

433

434

435

436

437

438

Mitchell, D.A., Docile, H.W., Greenfield, P.F., 1988. Agar plate growth studies of Rhizopus oligosporus and Aspergillus oryzae to determine their suitability for solid-state fermentation. Appl. Microbiol. Biotechnol. 598-602.

Pandey, A., Soccol, C.R., Larroche, C., 2008. Current Developments in Solid-state Fermentation. Springer New York. https://doi.org/10.1007/978-0-387-75213-6

Plavcan, J.M., 2004. A simple method for measuring colour in wild animals: Validation and use on chest patch colour in geladas (Theropithecus gelada). Sex. Sel. Primates New Comp. Perspect. 94, 230-252. https://doi.org/10.1111/j.1095-8312.2008.00981.x

Ramana Murthy, M.V., Thakur, M.S., Karanth, N.G., 1993. Monitoring of biomass in solid state fermentation using light reflectance. Biosens. Bioelectron. 8, 59-63. https://doi.org/10.1016/0956-5663(93)80044-P

Reeslev, M., Kjøller, A., 1995. Comparison of Biomass Dry Weights and Radial Growth Rates of Fungal Colonies on Media Solidified with Different Gelling Compounds. Appl. Environ. Microbiol. 61, 4236-4239.

Simeng, Z., Sacha, G., Isabelle, H.-G., Marie-Noëlle, R., 2015. A PCR-based method to quantify fungal growth during pretreatment of lignocellulosic biomass. J. Microbiol. 
448 Steudler, S., Bley, T., 2015. Biomass estimation during macro-scale solid-state fermentation of 449 basidiomycetes using established and novel approaches. Bioprocess Biosyst. Eng. 38, $450 \quad$ 1313-1323. https://doi.org/10.1007/s00449-015-1372-0

451 Stevens, M., Párraga, C.A., Cuthill, I.C., Partridge, J.C., Troscianko, T., 2007. Using digital 452 photography to study animal coloration. Biol. J. Linn. Soc. 90, 211-237. 453 https://doi.org/10.1111/j.1095-8312.2007.00725.x 


\section{Supplementary material}

456 As seen in Figure A, variations in density can occur even within the same colony.

457

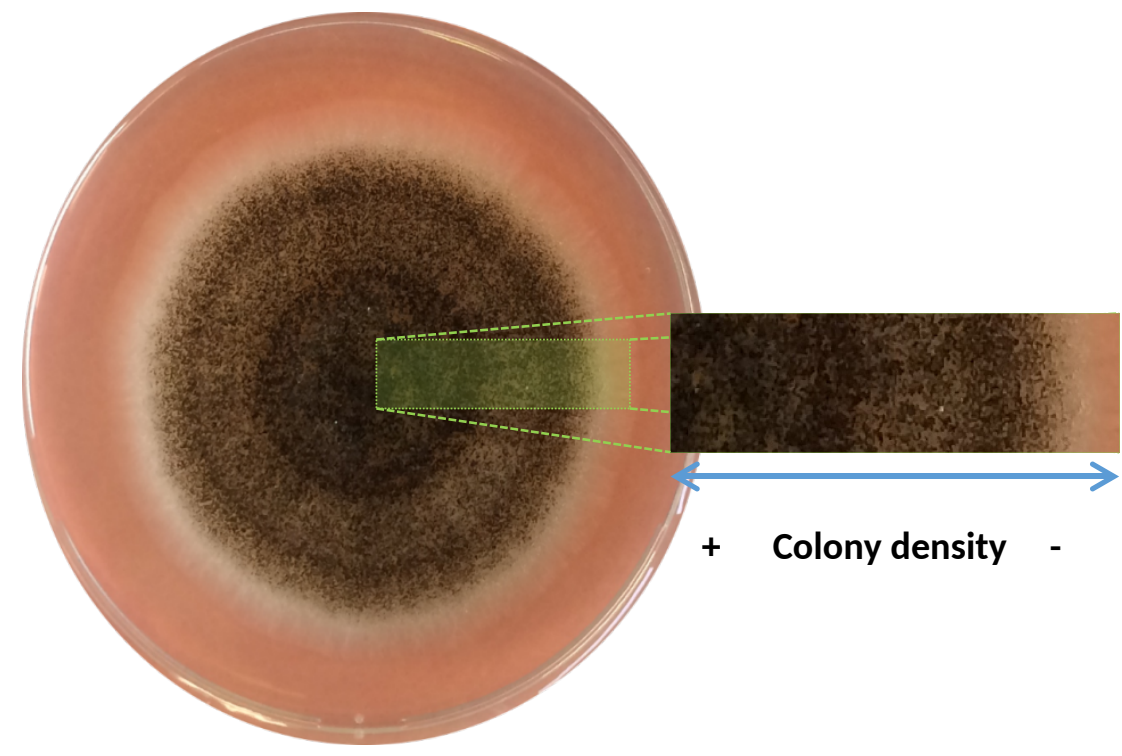

Figure A: Image of a petri dish with a colony of Aspergillus awamori. The petri dish was placed on an orange background to allow a better contrast between the colours. It is possible to observe that there is a difference between the fungal densities in different sections of the occupied area.

Figure A shows a colony of Aspergillus awamori growing on a potato dextrose agar (PDA) plate. The plate was placed on an orange background for a better contrast of the colours. The section without growth shows a uniform colour (orange in this case) and occupied areas show the colour of the fungal spores. Areas nearer to the centre of the plate show an apparent greater fungal density than those from the outer sections of the colony. This variation in colony density results in a perceivable difference between the colours in the image. Therefore, it should be possible, in principle, to use variations in the colour as an indicator of growth.

\section{Theory of the quantification of colour}

The human eye is able to detect colour thanks to the cone cells present in the retina. While there are millions of cone cells in the eye, they can be categorised into three types according to the wavelength (long, medium and short) that they are sensitive to. The colour that we perceive depends on the composition of wavelengths that enter the eye. For example, if most of the light 
473 that enters the eye has a wavelength of around $700 \mathrm{~nm}$ we see red and if it has a wavelength of

$474400 \mathrm{~nm}$ we perceive violet (x-rite, 2013).

475 The Commission Internationale de l'éclairage (CIE)

476 developed, in 1931 ,the 'CIE colour spaces' which were

477 the first defined quantitative links between physical pure

478 colours (i.e. wavelengths) in the electromagnetic visible

479 spectrum, and physiological perceived colours in

480 human colour vision (Hunt and Pointer, 2011). They

481 created a scale in which each colour has a value and is

482 obtained by adding different amounts of intensities of the

primaries red, green and blue (RGB), just as it happens in

484 the human eye. In this way, the combination of colours

485 that can be produced span in a space known as the RGB

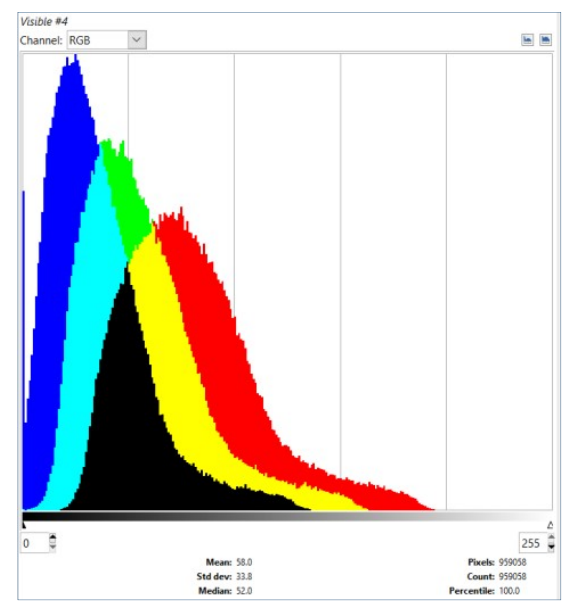

Figure B: Example of a colour histogram. The $x$-axis represents the colours, with various intensities, and the $y$-axis the number of pixels. Additionally, GIMP provides values for the total number of pixels, the mean, the median and the standard deviation. colour space (Dörge et al., 2000). Images produced for example in photography, video and computer monitors, are comprised of pixels. Each single pixel has a colour, defined by the RGB colour space system. Hence, it is possible to represent the colour distribution of any image using numerical values on a graph. This graph is known as a colour histogram (Figure B) which is a bar chart that shows the number of pixels for every specific colour of an image. 

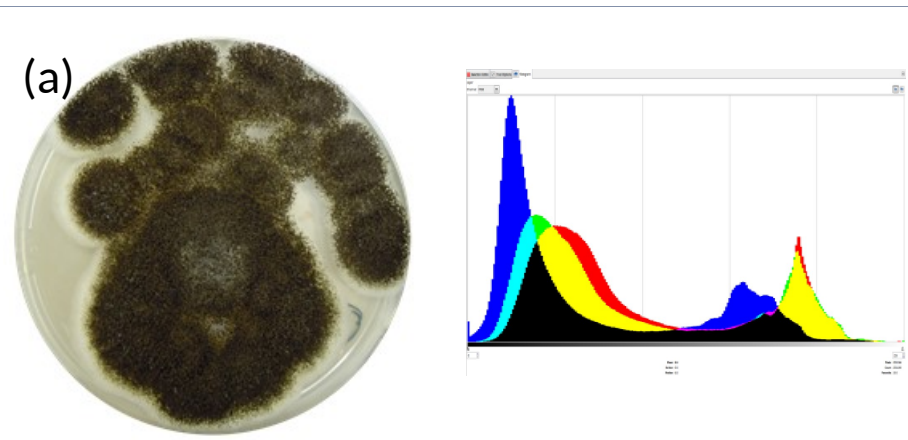

\begin{tabular}{|cc|}
\hline Pixels & Colour mean \\
20525207 & 88.5 \\
\hline
\end{tabular}
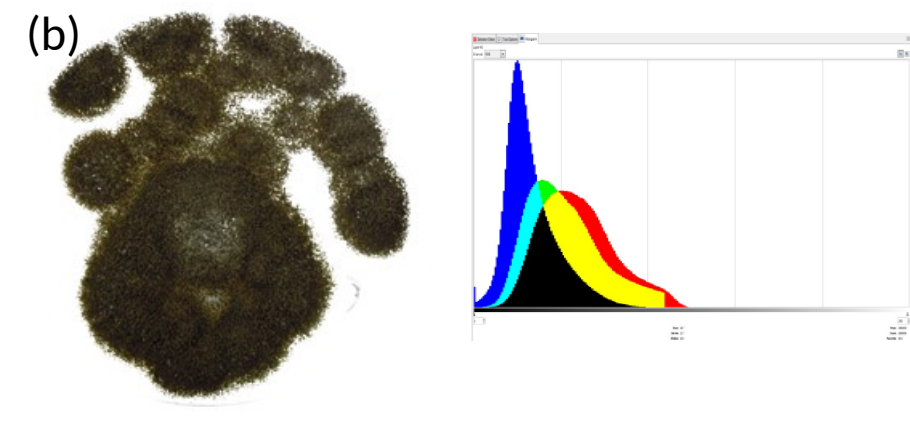

\begin{tabular}{cc} 
Pixels & Colour mean \\
14217738 & 50.2 \\
\hline
\end{tabular}

$$
X_{g}=\frac{14217738}{20525207}=0.69
$$

Figure C: Measuring colour intensity using GIMP software. (a) Image of a PDA plate with growth of $A$. awamori after $48 \mathrm{~h}$ of incubation at $30^{\circ} \mathrm{C}$. (b) Occupied area of the plate (69\% of the total). The software allows the measurement of the area, even when it is not regular, and the measurement of colour. As observed the (b) histogram is just a section of the (a) histogram which corresponds to the occupation area.

As observed in Figure $C$ the software selectively separates the occupied area (69\%), in spite of its irregular shape, and gives a value for the colour mean. As seen in the figure, after selecting the occupied area, the corresponding bars for the area free of fungi disappear from the histogram. Those bars belong to sections of the plate that have the colour of the agar and not the fungi. Furthermore, the colour mean of the area of fungi is 50.2 and the colour mean of the full plate is 88.5 . An agar plate without any growth would have a colour mean higher than those two values.

\section{Comparison of grayscale and colour images using DIA}

505 Figure D shows the mean values obtained from the same image in colour and greyscale. The 506 points were obtained from the images of the plates at 4 and 6 days shown in Figure 12. In both 507 set of samples, 4 and 6 days, results for the mean were lower for the grayscale images, with apparent larger differences with lower mean values. 


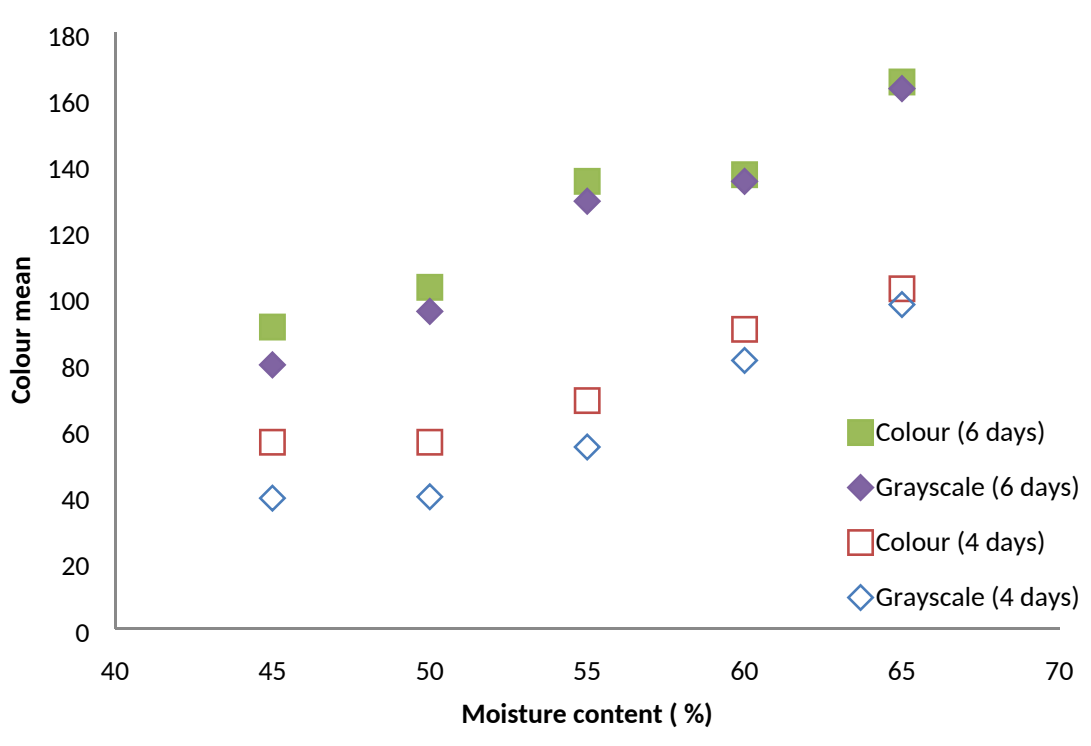

$510 \quad$ Figure D: Values for the means in colour and greyscale images of the plates from Figure 12.

511

512

513

514

515

516

517

518

519

520

521 